\title{
Bladder resident macrophages: Mucosal sentinels
}

Livia Lacerda Mariano ${ }^{1,2}$ and Molly A. Ingersoll ${ }^{1,2^{*}}$

${ }^{1}$ Unit of Dendritic Cell Immunobiology, Department of Immunology, Institut Pasteur, Paris France 75015

${ }^{2}$ Inserm U1223, Paris, France 75015

\section{*Corresponding author:}

Molly A. Ingersoll

Institut Pasteur

25 Rue du Docteur Roux

75015 Paris

+33(0)14568 8071

molly.ingersoll@pasteur.fr

Key words

bladder; urinary tract infection; bladder cancer; tissue-resident macrophage, monocytes; uropathogenic E. coli; BCG immunotherapy; Schistosoma haematobium 


\begin{abstract}
Macrophages are instrumental in the response to infectious and noninfectious diseases, however, their role in the bladder is poorly understood. Indeed, the bladder is a mucosal tissue frequently overlooked in research, despite the prevalence of illnesses such as urinary tract infection and bladder cancer. Notably, bladder tissue macrophages are among the most populous resident immune cells in this organ and recent studies support that resident macrophages and infiltrating monocytes play nonredundant roles in response to infection, immunotherapy, and inflammation. Advancing our understanding of macrophage behavior in the bladder is complicated by the difficulty in obtaining tissue-resident cells. Surmounting this challenge, however, for a greater understanding of macrophage ontology, impact on innate and adaptive immunity, and regulation of homeostasis, will ultimately contribute to better therapies for common afflictions of the bladder.
\end{abstract}




\section{THE BLADDER}

The bladder is an expandable hollow organ located in the pelvic area. It is part of the urinary tract system, which includes the kidneys, ureters, and urethra. Its main function is to store urine produced in the kidneys prior to voiding [1]. Despite its apparent simplicity, the bladder has a complex morphology with multiple cell types, including the most impenetrable epithelium of the body [2]. This feature is vital to protect host tissues from toxins accumulated in the urine and to prevent invasion of microorganisms [3]. Structurally, a strong muscular wall, the lamina propria, and a layer of adipose tissue surround and support the epithelial layer in the bladder, the urothelium [4]. The urothelium is composed of three to six layers of cells. The basal layer is most proximal to the lamina propria, followed by intermediate cells, which make up one or more layers of cells. Very large binucleated cells, called umbrella or facet cells, make up the superficial layer.

The urinary tract is particularly vulnerable to infection as the urethral opening is in close proximity to the terminus of the gastrointestinal tract. As such, the bladder has multiple mechanisms of host protection [5]. The immune cell compartment is well characterized in naïve mouse bladders [6]. It is primarily composed of phagocytic antigen presenting cells, such as dendritic cells and macrophages [6]. Additional resident immune cells include $\mathrm{CD}^{+}$and $\gamma \delta^{+} \mathrm{T}$ cells, NK cells, monocytes, eosinophils, and mast cells [6]. Innate lymphoid cells are also likely resident in the bladder, however additional studies are needed to address this possibility. Much less is known about the bladder-resident immune

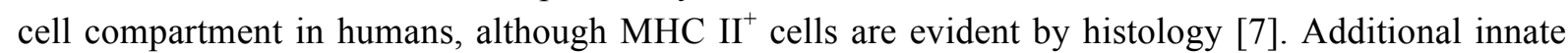
bladder defense mechanisms include i) micturition, which may act to impede bacterial ascension or colonization, but appears to facilitate adhesion for certain uropathogens; ii) a mucin layer that impedes bacterial adherence to the urothelium; and iii) soluble factors, such as complement and antimicrobial peptides, immunoglobulin A, Tamm-Horsfall protein, and iron sequestering lactoferrin and lipocalin $[5,8-11]$.

Finally, similar to other anatomical sites, the bladder harbors a unique microflora, which is distinct from the gut and vagina microbiomes, and is not sterile, as previously thought [12-17]. The urinary microbiome appears to differ between men and woman. Women may have a more diverse urinary microbiome than men, however, the very small number of individuals sampled in this study precludes making broad generalizations about microbial diversity [16]. Urinary diseases, such as infection and catheterization can disrupt the healthy microbiome in men and women, leading to transient or longterm changes in its composition $[16,17]$. While more studies are needed, the urinary microbiota may prevent colonization of the bladder by uropathogens through competition for resources or niches or may more generally support urinary tract health. Supporting this hypothesis, alterations in the microflora have been linked to non-infectious urological diseases, such as interstitial cystitis [18-20].

\section{DISEASES OF THE BLADDER}

Urinary tract infection (UTI) is one of the most common infections in humans and is the most common hospital-acquired infection [21-23]. Uropathogenic Escherichia coli (UPEC) is the causative agent in more than $80 \%$ of UTI [24]. Other uropathogens include, but are not limited to Klebsiella pneumoniae, Enterococcus faecalis, Staphylococcus saprophyticus, and Proteus mirabilis [25-27]. UTI exhibits a sex-bias, in which women are more susceptible to infection than men. Indeed, approximately $50 \%$ of all women will experience a UTI, and approximately one-fourth to one half of these woman will have a recurrent infection within 6-12 months [28]. Susceptibility to UTI may be determined by genetic factors, functional abnormalities of the urinary tract, use of catheters, or 
composition of the urinary microbiota $[29,30]$, although these factors cannot entirely explain the prevalence of infection. Genetic predisposition appears to be a major factor in UTI morbidity, as individuals with a family history of UTI are at increased risk of developing acute cystitis [23, 29, 31, 32].

Bladder cancer is the ninth most common malignancy in the world and is much more common in men than in women [33-35]. Nonetheless, women are more likely to have locally advanced tumors and worse outcomes compared to male patients [34]. Exposure to carcinogens, including those in cigarette smoke, is a major risk factor for developing bladder cancer [36]. Additional risk factors include exposure to arsenic and occupational exposure to chemicals, such as aromatic amines and 2chloroaniline [37]. Bladder cancer can be subdivided into nonmuscle and muscle invasive disease, with distinct treatments and outcomes associated with each [33]. In addition, a distinct form of squamous cell carcinoma develops in the bladder following chronic bladder infection by Schistosoma haematobium [38]. Schistosoma haematobium is endemic in Egypt and other parts of Sub-Saharan Africa, making squamous cell carcinoma the predominant form of bladder cancer in this part of the world [39-41].

Lastly, the bladder can be affected by a relatively poorly understood disease called interstitial cystitis (IC). IC or painful bladder syndrome (PBS) is a sterile chronic inflammatory condition primarily occurring in women. IC/PBS symptoms include the frequency and urgency to urinate, and discomfort and pain in the bladder and pelvic region [42]. IC/PBS is poorly defined and clinical symptoms are shared with other diseases, such as irritable bowel syndrome and fibromyalgia, which makes diagnosis and treatment challenging [43]. The etiology of the disease is also unknown. However, one possibility is that symptoms arise following an inappropriate inflammatory response to an infection [44, 45]. As the bladder tends to be overlooked and research in bladder diseases is severely underfunded [46], there is still a large gap in our knowledge of specific mucosal immune responses following inflammation, infection, and cancer. This translates to limited therapeutic options for these bladder diseases.

\section{THE ROLE OF MACROPHAGES DURING URINARY TRACT INFECTION}

Bacterial ascension into the urinary tract may lead to infection. Once invading bacteria reach the bladder mucosa, they can induce a strong inflammatory response arising from nonimmune and immune cells [8]. The presence of phagocytes and antigen presenting cells in the bladder suggests these cells play an important role in immune surveillance locally. Macrophages comprise nearly $40 \%$ of all CD $45^{+}$cells in the bladder immune cell compartment of mice [6]. Consequently, they contribute to the host immune response to UTI in several ways.

\section{Recognizing uropathogens}

The host inflammatory response to infection is initiated upon innate bacterial sensing by pattern recognition receptors (PRR). Notably, many PRR, such as Toll-like receptors (TLR)2, TLR4, TLR5, TLR11 are expressed in the bladder [8]. Although these receptors are typically associated with macrophages, dendritic cells, or epithelial cells, their expression patterns in the bladder have not been mapped to specific cell types. Among the various PRR, TLR are the best characterized in the context of UTI. Components of UPEC, such as type I pili, activate TLR4, for example [47]. TLR4 signaling from both the hematopoietic and stromal compartments is critical to mount an effective immune response against UPEC [48]. Moreover, variation in the promoter of the TLR4 gene is associated with human UTI susceptibility [49], suggesting that TLR4 plays a protective role in UTI. In addition to TLR4, CD14, an LPS receptor, can be detected during UPEC infection, and its loss leads to a modest 
increase in bacterial burden and an aberrant transcriptional signature in the bladder in experimental UTI [50]. Finally, mice lacking TLR5, which recognizes bacterial flagellin, have increased bacterial burden and inflammation 5 days post-infection compared to wild-type controls [51].

\section{Inducing inflammation}

Inflammation is critical to induce recruitment of immune cells that subsequently kill uropathogens, and while macrophages are likely an important source of pro-inflammatory cytokines during UTI [52]. In addition to PRR activation, production of pro-inflammatory cytokines follows bacterial recognition through intracellular receptors such as inflammasomes [53, 54]. For example, inflammasome activation leads to the release of proinflammatory mediators, such as IL-1 $\beta$, and UPEC strains can induce the NLRP3 inflammasome in mouse models of UTI [54]. Furthermore, inflammasome activation is enhanced in ATG16L1 hypomorphic mouse bone marrow derived macrophages infected with UPEC, leading to increased IL-1 $\beta$ secretion [55]. Accordingly, infected ATG16L1 hypomorphic mice have improved bacterial clearance compared to wildtype animals [55]. A specific phylogenetic group of UPEC expresses a virulence factor, Toll/IL-1 receptor-containing (TIR-containing) protein C $(\mathrm{TcpC})$, that, in mouse bone marrow derived macrophages, interacts with the NLRP3 inflammasome to reduce caspase- 1 activation and IL-1 $\beta$ release compared to UPEC deficient in this protein [54]. In vivo, mice infected with TcpC deficient-UPEC have reduced levels of IL-1 $\beta$ in the urine compared to mice infected with wildtype bacteria [54]. A number of different UPEC strains associated with symptomatic UTI induce inflammasome activation, IL-1 $\beta$ release, and cell death to different degrees in human monocyte-derived macrophages and mouse bone marrow derived macrophages [56]. Inflammasome responses in mouse macrophages are largely dependent on the UPEC virulent factor $\alpha$ hemolysin, while cell death and IL-1 $\beta$ secretion in human macrophages occur independently from this toxin [56]. This divergence in response between human and mouse macrophages suggests that UPEC may be differently recognized, resulting in the activation of distinct pathways, depending on the host cell species. Alternatively, these differences may reflect studies performed in vitro in macrophages matured by different means.

Despite the cellular machinery fighting infection, bacteria may subvert the immune system to persist in the host. One study suggests that UPEC subverts macrophage killing and survives inside bone marrow derived macrophages up to 24 hours [57]. However, as the number of bacteria recovered 24 hours post-infection in this study was much lower than the initial bacterial input, it is difficult to conclude that UPEC can survive inside macrophages, particularly with a single time point measurement [57]. Alternatively, it may be that bacteria do not necessarily survive, but are not entirely killed at 24 hours post-infection. Additional time points, uptake and degradation studies, and in vivo experiments are needed to determine whether UPEC can truly persist inside macrophages. It is noteworthy that only one study has investigated UPEC infection using macrophages isolated from the bladder [58]. In this study, macrophages isolated from mouse bladders upregulate iron sequestering molecules, thereby likely limiting iron availability for UPEC and inhibiting growth in the bladder [58]. By contrast, the vast majority of in vitro studies investigating macrophage-UPEC interactions rely upon monocyte-derived or peritoneal macrophages. These macrophages are widely used to perform in vitro studies given the ease in obtaining high number of cells. However, they do not truly resemble tissue resident macrophages, which, in most tissues have an embryonic origin rather than bone marrow [59]. In addition, tissue microenvironment crosstalk mediates transcriptional changes in resident macrophages, and thus macrophage response would be expected to have at least some unique features in each tissue [60]. Thus, while bone marrow or monocyte-derived cells provide a valuable in vitro model, it is clear that they cannot entirely reflect the biology of tissue resident macrophages and great 
care must be taken when extrapolating findings from these systems to tissue-specific macrophage behavior in vivo [61, 62].

\section{Mediating innate immune responses}

A hallmark feature of infection is the recruitment of inflammatory cells, such as neutrophils and monocytes $[63,64]$. Neutrophils are potent phagocytes and are recruited in high numbers to the bladder during UTI, where they take up bacteria and contribute to clearance [65]. Depletion of G-CSF, a key factor for neutrophil survival and differentiation, results in decreased numbers of neutrophils in circulation and reduced infiltration into the bladder during UTI [66]. Surprisingly, however, reduced neutrophil infiltration results in a lower bacteria burden in infected bladders. This result suggests that other cell types, such as monocytes or macrophages, are also critical for UPEC clearance [66]. This conclusion is supported by the finding that depletion of both neutrophils and classical monocytes, by targeting Ly6C and Ly6G with the Gr-1 antibody [67], results in a much higher bacterial burden compared to control-treated mice [65]. Indeed, upon infection, monocytes robustly infiltrate the bladder early during infection $[6,52,63,66]$. Monocytes are likely attracted to the bladder by the expression of several chemokines, such as CCL2. Supporting this, the abundance of Ly6C ${ }^{+} \mathrm{CD} 115^{+}$ cells in the bladder is reduced by approximately $50 \%$ following anti-CCL2 antibody treatment [52].

Tracing the fate of infiltrating bead-labeled monocytes revealed that a vast majority of these cells differentiate into cells resembling tissue resident macrophages, whereas a small subset $(\sim 10 \%)$ differentiates into dendritic cells [6]. It has been suggested that during UTI, resident macrophages, defined as lacking the protein Ly6C, produce chemokines, such as CXCL1 and CCL2, to attract neutrophils and $\mathrm{Ly} 6 \mathrm{C}^{+}$classical monocytes, respectively, from circulation [52]. Once in the tissue, these monocyte-derived macrophages produce TNF- $\alpha$, which induces release of CXCL2 from Ly6C ${ }^{-}$ resident macrophages. Subsequently, CXCL2 expression leads to the release of matrix metalloproteinase 9 by neutrophils, allowing them to penetrate infected tissue and phagocytize bacteria [52]. These findings suggest that monocytes may infiltrate infected bladder tissue before neutrophils. As it has also been reported that neutrophils enter the bladder prior to monocytes [66], additional kinetic studies may be justified. In addition, in at least one study, bladder macrophage depletion does not lead to a reduction in chemokine expression, including CCL2 or CXCL1, during infection [6]. Therefore, this is likely not the only mechanism to recruit neutrophils and monocytes, especially considering that urothelial-derived chemokines also mediate neutrophil infiltration $[68,69]$.

Contrasting roles for macrophages in UPEC uptake have also been observed. One study, from Schiwon and colleagues, reported that while macrophages play a role in recruiting immune cells, they themselves do not take up UPEC [52]. By contrast, another study, from Mora-Bau and colleagues, observed that macrophages are the principal antigen-presenting cell to acquire bacteria early during infection [6]. The discrepancy between these two reports can best be explained by the use of different fluorescent-expressing UPEC. While the Schiwon study used a green fluorescent protein (GFP)expressing bacterial strain, the Mora-Bau study used a red fluorescent protein (RFP)-expressing UPEC

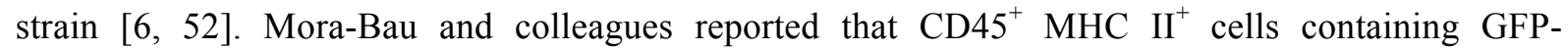
expressing UPEC were masked by abundant autofluorescence in the bladder, arising from the urothelium and uninfected bladder resident macrophages [6]. Using an RFP UPEC strain, Mora-Bau and colleagues identified infected cells, including tissue resident macrophages and monocytes among the naturally autofluorescent cell populations [6].

\section{Shaping adaptive immunity}


Macrophages do not only play a role in the innate response, but also impact development of adaptive immunity. Depletion of resident macrophages, prior to primary UTI, significantly improves bacterial clearance after a challenge infection with an isogenic UPEC strain [6]. Improved bacterial clearance following macrophage depletion is lost when lymphocytes are absent or when $\mathrm{T}$ cells are depleted, demonstrating that the phenotype is dependent upon components of the adaptive immune system [6]. In the absence of macrophages, dendritic cells take up significantly more bacteria, which suggests that resident macrophages negatively impact development of adaptive immunity in the bladder by sequestering bacteria from dendritic cells, or potentially by other means [6]. It remains to be demonstrated whether increased antigen uptake by dendritic cells leads to enhanced antigen presentation in the absence of macrophages.

Resident macrophage depletion does not appear to impact bacterial clearance during the primary infection following resident macrophage depletion [6]. However, a second study reported that macrophage depletion leads to a modestly higher bacterial burden 24 hours post-infection [50]. These conflicting results can likely be explained by the different methods used to deplete macrophages. Mora-Bau and colleagues used an anti-CD115 antibody to deplete resident macrophages, as previously established [70], and confirmed that macrophages are depleted in the bladder by flow cytometry. By contrast, Carey and colleagues used clodronate liposomes [50], which deplete monocytes from circulation but do not impact the number of tissue-resident macrophages in the bladder [6]. The decrease in macrophage number, reported by Carey and colleagues after infection, likely reflects a decrease in monocyte infiltration due to clodronate depletion of these cells in circulation [50]. Timing of depletion and route of injection may also underlie the differing findings, as Mora-Bau et al. reported that a reduction in circulating monocytes, by clodronate treatment or in the CCR $2^{-/}$mouse, did not lead to increased bacterial CFU following infection [6]. While Mora-Bau and colleagues administered clodronate 15-18 hours prior to infection via intravenous injection [6], Carey et al. injected clodronate intraperitoneally 24 hours and 2 hours before infection [50]. Importantly, these divergent findings stress the importance of analyzing the target organ, additional tissues such as blood circulation, and non-targeted cells, prior to or at the time of infection to have a complete understanding of the environment during infection.

\section{MACROPHAGES IN THE CONTEXT OF BLADDER CANCER AND THERAPY}

The standard of care treatment for high-risk nonmuscle invasive bladder cancer is intravesical instillation of an attenuated form of Mycobacterium bovis, Bacillus Calmette-Guérin (BCG), following transurethral resection [71]. BCG immunotherapy reduces disease recurrence and progression in at least $50 \%$ of cases $[71,72]$. Following instillation of BCG directly into the bladder, an array of cytokines can be detected in the urine of patients [73-75]. BCG intravesical therapy also induces a significant infiltration of inflammatory cells, indispensable for the success of this immunotherapy, such as neutrophils, monocytes, and lymphocytes [76-79]. Although the mechanisms underlying BCG treatment are not completely understood, it is well accepted that the anti-tumor effect of BCG treatment is through the activation of the immune system and induction of an inflammatory response leading to lasting immune-mediated protection [76].

Macrophages accumulate in tumors with differing consequences [78]. However, little attention has been paid to macrophages in the context of bladder cancer and immunotherapy. Whether and how macrophages contribute to tumor eradication or increased vascularization, tumor growth, and metastasis is not entirely clear. Macrophages can be found in the urine and bladder wall of patients treated with BCG intravesically [80]. In vitro, peritoneal macrophages can kill the bladder cancer cell 
line, MBT-2, and enhanced cytotoxicity is correlated with increased TNF- $\alpha$, IFN- $\gamma$, and IL-12 release by macrophages when stimulated with BCG [80, 81]. Macrophages from C57B1/6 mice are less cytotoxic to bladder cancer cells than macrophages derived from $\mathrm{C} 3 \mathrm{H} / \mathrm{HeN}$ mice, and this reduced cytotoxicity is correlated with production of high levels of IL-10 by $\mathrm{C} 57 \mathrm{Bl} / 6$ macrophages [82]. Additionally, macrophages from IL-10 knockout mice are more cytotoxic against MB49 cells as compared to wildtype macrophages [82]. Importantly, these in vitro studies relied upon peritoneal exudate as a source for macrophages, again limiting translation to bladder-resident macrophages or tumor macrophages.

Those studies that have addressed the impact of tumor-associated macrophages on bladder cancer prognosis have concluded that macrophage presence in tumors, prior to BCG therapy, correlates with an increased risk for recurrence [83-85]. Furthermore, a predominance of M2 activated macrophages, which support development of $\mathrm{T}_{\mathrm{h}}$ 2-skewed immunity, is associated with $\mathrm{BCG}$ therapy failure [86]. These observations support the conclusion that BCG therapy requires a $T_{h} 1$ biased immune response for effective tumor immunity and are in line with in vitro results demonstrating that the $\mathrm{T}_{\mathrm{h}} 1$ cytokines TNF- $\alpha$, IFN- $\gamma$, and IL-12 are associated with increased macrophage cytotoxicity [87] [80, 81]. These interpretations, however, are complicated by the methods used to identify macrophages and the "increased risk" cutoff values, which vary widely for each study. In addition, although these studies suggest that macrophages play a negative role in the tumor immune response to BCG therapy, only a subset of M2-like activated macrophages were considered [86]. Whether other subpopulations of tumor associated macrophages, deriving from resident macrophages or infiltrating monocytes, may be beneficial or play competing roles during BCG therapy remains to be investigated.

Finally, little is known about the role of macrophages in bladder cancer induced by chronic infection with Schistosoma haematobium. Histopathologic analysis in the bladder of mice with urinary tract infection induced by $S$. haematobium reveals the presence of macrophages in granulomas in the bladder wall [88]. Chi-Ling Fu and colleagues suggest that clodronate-mediated monocyte depletion in experimental urogenital schistosomiasis leads to increased mortality, highlighting the importance of these cells in host protection in S. haematobium-induced urogenital infection [89]. Importantly, however, no studies have yet demonstrated a clear role for macrophages in bladder cancer progression resulting from urogenital $S$. haematobium infection. Indeed, very little is known about the role of macrophages in this particular bladder malignancy and further investigation is needed.

\section{WHAT REMAINS TO BE DISCOVERED}

One of the biggest challenges in working with bladder macrophages in preclinical animal models, such as mice, is the relatively small size of this organ and the minute number of macrophages that can be recovered [6]. This challenge certainly contributes to the reliance on bone marrow derived or peritoneal macrophages. However, it is critical to take into consideration the biological differences among different tissue-resident macrophages and how these differences impact macrophage response in various diseases [60].

As an example, in UTI and bladder cancer, specific macrophages subsets (e.g., monocyte derived or resident) may be supportive or detrimental to bladder immunity, impacting the strength of the adaptive response [6]. We can speculate that tissue-resident macrophages may negatively impact bladder immunity through polarization to M2-like immune suppressive cells and targeting these antiinflammatory macrophages or boosting the inflammatory response to bacteria or tumors with cytokines or recombinant bacteria, such as UPEC or BCG, may help to overcome this issue. 
We ask whether macrophages in the bladder are a necessary evil. This is because we have evidence that they may negatively impact immunity in disease states [6]. The mechanisms, however, by which macrophages impact bladder immunity and tissue integrity are not well understood and more studies are required to elucidate the role of bladder macrophages in both homeostasis and disease. In other tissues, macrophages contribute to organ development, homeostasis, and repair [90]. Across diverse tissues, repair after injury occurs in overlapping phases of inflammation, proliferation, and remodeling. Macrophages, in particular, are essential for regeneration, repair, and remodeling in numerous tissues [91-94]. Critically, almost nothing is known about how macrophages are maintained or replaced in the bladder after injury or the role of macrophages in facilitating the return to tissue homeostasis in the bladder. The bladder urothelium undergoes constant membrane recycling [95] and may require the support of macrophages in maintaining its tight barrier function. Finally, to truly understand these cells, we must discern the origin of resident macrophages and how inflammation and/or infection impacts macrophage populations, their renewal, and their function in the bladder over time. Spatial localization, activation status, and cytokine expression profiles will elucidate the relationship of this cell with other immune and stromal cells in homeostasis, infection, and cancer. Future studies along these lines will contribute to the development of new, targeted immunotherapies for infectious and noninfectious diseases of the bladder.

\section{ACKNOWLEDGEMENTS}

The authors would like to thank Conan O'Brien and Drs Alba Llibre and Björn Albrecht for insightful comments and critical review of this manuscript. LLM is part of the Pasteur-Paris University International $\mathrm{PhD}$ Program. This program has received funding from the European Union's Horizon 2020 research and innovation program under the Marie Sklodowska-Curie grant agreement No. 665807 and from the Labex Milieu Intérieur project at Institut Pasteur, which is part of the French government's Invest in the Future Program (LabEx), managed by the Agence Nationale de la Recherche. Funding was also provided by the LabEx Immuno-Onco project (LLM and MAI). 


\section{REFERENCES}

[1] W.C. de Groat, Anatomy and physiology of the lower urinary tract, Urol Clin North Am, 20 (1993) 383-401.

[2] H.O. Negrete, J.P. Lavelle, J. Berg, S.A. Lewis, M.L. Zeidel, Permeability properties of the intact mammalian bladder epithelium, Am J Physiol, 271 (1996) F886-894.

[3] K.E. Sivick, H.L. Mobley, Waging war against uropathogenic Escherichia coli: winning back the urinary tract, Infect Immun, 78 (2010) 568-585.

[4] R.M. Hicks, The mammalian urinary bladder: an accommodating organ, Biol Rev Camb Philos Soc, 50 (1975) 215-246.

[5] M.A. Ingersoll, M.L. Albert, From infection to immunotherapy: host immune responses to bacteria at the bladder mucosa, Mucosal Immunol, 6 (2013) 1041-1053.

[6] G. Mora-Bau, A.M. Platt, N. van Rooijen, G.J. Randolph, M.L. Albert, M.A. Ingersoll, Macrophages Subvert Adaptive Immunity to Urinary Tract Infection, PLoS Pathog, 11 (2015) e1005044.

[7] R.A. Gardiner, G.J. Seymour, M.F. Lavin, G.M. Strutton, E. Gemmell, G. Hazan, Immunohistochemical analysis of the human bladder, Br J Urol, 58 (1986) 19-25.

[8] S.N. Abraham, Y. Miao, The nature of immune responses to urinary tract infections, Nat Rev Immunol, 15 (2015) 655-663.

[9] W.E. Thomas, E. Trintchina, M. Forero, V. Vogel, E.V. Sokurenko, Bacterial adhesion to target cells enhanced by shear force, Cell, 109 (2002) 913-923.

[10] E. Miller, T. Garcia, S. Hultgren, A.F. Oberhauser, The mechanical properties of E. coli type 1 pili measured by atomic force microscopy techniques, Biophys J, 91 (2006) 3848-3856.

[11] J. Zakrisson, K. Wiklund, O. Axner, M. Andersson, The shaft of the type 1 fimbriae regulates an external force to match the FimH catch bond, Biophys J, 104 (2013) 2137-2148.

[12] L. Brubaker, A.J. Wolfe, The female urinary microbiota, urinary health and common urinary disorders, Ann Transl Med, 5 (2017) 34.

[13] L. Brubaker, A.J. Wolfe, Microbiota in 2016: Associating infection and incontinence with the female urinary microbiota, Nat Rev Urol, 14 (2017) 72-74.

[14] E.E. Hilt, K. McKinley, M.M. Pearce, A.B. Rosenfeld, M.J. Zilliox, E.R. Mueller, L. Brubaker, X. Gai, A.J. Wolfe, P.C. Schreckenberger, Urine is not sterile: use of enhanced urine culture techniques to detect resident bacterial flora in the adult female bladder, J Clin Microbiol, 52 (2014) 871-876.

[15] D.A. Lewis, R. Brown, J. Williams, P. White, S.K. Jacobson, J.R. Marchesi, M.J. Drake, The human urinary microbiome; bacterial DNA in voided urine of asymptomatic adults, Front Cell Infect Microbiol, 3 (2013) 41.

[16] D.E. Fouts, R. Pieper, S. Szpakowski, H. Pohl, S. Knoblach, M.J. Suh, S.T. Huang, I. Ljungberg, B.M. Sprague, S.K. Lucas, M. Torralba, K.E. Nelson, S.L. Groah, Integrated next-generation sequencing of $16 \mathrm{~S}$ rDNA and metaproteomics differentiate the healthy urine microbiome from asymptomatic bacteriuria in neuropathic bladder associated with spinal cord injury, J Transl Med, 10 (2012) 174.

[17] C. Lassek, M. Burghartz, D. Chaves-Moreno, A. Otto, C. Hentschker, S. Fuchs, J. Bernhardt, R. Jauregui, R. Neubauer, D. Becher, D.H. Pieper, M. Jahn, D. Jahn, K. Riedel, A metaproteomics approach to elucidate host and pathogen protein expression during catheter-associated urinary tract infections (CAUTIs), Mol Cell Proteomics, 14 (2015) 989-1008.

[18] S.A. Whiteside, H. Razvi, S. Dave, G. Reid, J.P. Burton, The microbiome of the urinary tract--a role beyond infection, Nat Rev Urol, 12 (2015) 81-90.

[19] H. Siddiqui, K. Lagesen, A.J. Nederbragt, S.L. Jeansson, K.S. Jakobsen, Alterations of microbiota in urine from women with interstitial cystitis, BMC Microbiol, 12 (2012) 205.

[20] M.G. Abernethy, A. Rosenfeld, J.R. White, M.G. Mueller, C. Lewicky-Gaupp, K. Kenton, Urinary Microbiome and Cytokine Levels in Women With Interstitial Cystitis, Obstet Gynecol, 129 (2017) 500-506.

[21] B. Foxman, P. Brown, Epidemiology of urinary tract infections: transmission and risk factors, incidence, and costs, Infect Dis Clin North Am, 17 (2003) 227-241. 
[22] M.L. Wilson, L. Gaido, Laboratory diagnosis of urinary tract infections in adult patients, Clin Infect Dis, 38 (2004) 1150-1158.

[23] S. Esposito, V. Emmi, F.S. Mennini, F. Montorsi, G. Sganga, S. Leone, [Management and prevention of catheter-associated urinary tract infections: current opinions and clinical practice], Infez Med, 19 (2011) 74-90.

[24] G.C. Ulett, M. Totsika, K. Schaale, A.J. Carey, M.J. Sweet, M.A. Schembri, Uropathogenic Escherichia coli virulence and innate immune responses during urinary tract infection, Current opinion in microbiology, 16 (2013) 100-107.

[25] D.J. Farrell, I. Morrissey, D. De Rubeis, M. Robbins, D. Felmingham, A UK multicentre study of the antimicrobial susceptibility of bacterial pathogens causing urinary tract infection, J Infect, 46 (2003) 94-100.

[26] P.A. Jordan, A. Iravani, G.A. Richard, H. Baer, Urinary tract infection caused by Staphylococcus saprophyticus, J Infect Dis, 142 (1980) 510-515.

[27] J.N. Schaffer, M.M. Pearson, Proteus mirabilis and Urinary Tract Infections, Microbiol Spectr, 3 (2015).

[28] H.L. Mobley, C.J. Alteri, Development of a Vaccine against Escherichia coli Urinary Tract Infections, Pathogens, 5 (2015).

[29] G. Godaly, I. Ambite, C. Svanborg, Innate immunity and genetic determinants of urinary tract infection susceptibility, Curr Opin Infect Dis, 28 (2015) 88-96.

[30] V. Nienhouse, X. Gao, Q. Dong, D.E. Nelson, E. Toh, K. McKinley, P. Schreckenberger, N. Shibata, C.S. Fok, E.R. Mueller, L. Brubaker, A.J. Wolfe, K.A. Radek, Interplay between bladder microbiota and urinary antimicrobial peptides: mechanisms for human urinary tract infection risk and symptom severity, PLoS One, 9 (2014) e114185.

[31] D. Scholes, T.R. Hawn, P.L. Roberts, S.S. Li, A.E. Stapleton, L.P. Zhao, W.E. Stamm, T.M.

Hooton, Family history and risk of recurrent cystitis and pyelonephritis in women, J Urol, 184 (2010) 564-569.

[32] D. Scholes, T.M. Hooton, P.L. Roberts, A.E. Stapleton, K. Gupta, W.E. Stamm, Risk factors for recurrent urinary tract infection in young women, J Infect Dis, 182 (2000) 1177-1182.

[33] M. Burger, J.W. Catto, G. Dalbagni, H.B. Grossman, H. Herr, P. Karakiewicz, W. Kassouf, L.A. Kiemeney, C. La Vecchia, S. Shariat, Y. Lotan, Epidemiology and risk factors of urothelial bladder cancer, Eur Urol, 63 (2013) 234-241.

[34] J. Dobruch, S. Daneshmand, M. Fisch, Y. Lotan, A.P. Noon, M.J. Resnick, S.F. Shariat, A.R. Zlotta, S.A. Boorjian, Gender and Bladder Cancer: A Collaborative Review of Etiology, Biology, and Outcomes, Eur Urol, 69 (2016) 300-310.

[35] S. Antoni, J. Ferlay, I. Soerjomataram, A. Znaor, A. Jemal, F. Bray, Bladder Cancer Incidence and Mortality: A Global Overview and Recent Trends, Eur Urol, 71 (2017) 96-108.

[36] M.P. Zeegers, F.E. Tan, E. Dorant, P.A. van Den Brandt, The impact of characteristics of cigarette smoking on urinary tract cancer risk: a meta-analysis of epidemiologic studies, Cancer, 89 (2000) 630-639.

[37] S. Letasiova, A. Medve'ova, A. Sovcikova, M. Dusinska, K. Volkovova, C. Mosoiu, A. Bartonova, Bladder cancer, a review of the environmental risk factors, Environ Health, 11 Suppl 1 (2012) S11.

[38] M.H. Mostafa, S.A. Sheweita, P.J. O'Connor, Relationship between schistosomiasis and bladder cancer, Clin Microbiol Rev, 12 (1999) 97-111.

[39] M. Ploeg, K.K. Aben, L.A. Kiemeney, The present and future burden of urinary bladder cancer in the world, World J Urol, 27 (2009) 289-293.

[40] A.S. Felix, A.S. Soliman, H. Khaled, M.S. Zaghloul, M. Banerjee, M. El-Baradie, M. El-Kalawy, A.A. Abd-Elsayed, K. Ismail, A. Hablas, I.A. Seifeldin, M. Ramadan, M.L. Wilson, The changing patterns of bladder cancer in Egypt over the past 26 years, Cancer Causes Control, 19 (2008) 421-429. [41] M.J. van der Werf, S.J. de Vlas, S. Brooker, C.W. Looman, N.J. Nagelkerke, J.D. Habbema, D. Engels, Quantification of clinical morbidity associated with schistosome infection in sub-Saharan Africa, Acta Trop, 86 (2003) 125-139.

[42] S.S. Patnaik, A.S. Lagana, S.G. Vitale, S. Buttice, M. Noventa, S. Gizzo, G. Valenti, A.M.C. Rapisarda, V.L. La Rosa, C. Magno, O. Triolo, V. Dandolu, Etiology, pathophysiology and 
biomarkers of interstitial cystitis/painful bladder syndrome, Arch Gynecol Obstet, 295 (2017) 13411359.

[43] P. Hanno, J. Nordling, A. van Ophoven, What is new in bladder pain syndrome/interstitial cystitis?, Curr Opin Urol, 18 (2008) 353-358.

[44] I. Offiah, A. Didangelos, J. Dawes, R. Cartwright, V. Khullar, E.J. Bradbury, S. O'Sullivan, D. Williams, I.P. Chessell, K. Pallas, G. Graham, B.A. O'Reilly, S.B. McMahon, The Expression of Inflammatory Mediators in Bladder Pain Syndrome, Eur Urol, 70 (2016) 283-290.

[45] S. Grover, A. Srivastava, R. Lee, A.K. Tewari, A.E. Te, Role of inflammation in bladder function and interstitial cystitis, Ther Adv Urol, 3 (2011) 19-33.

[46] J.L. Boormans, E.C. Zwarthoff, Limited Funds for Bladder Cancer Research and What Can We Do About It, Bladder Cancer, 2 (2016) 49-51.

[47] H. Kumar, T. Kawai, S. Akira, Toll-like receptors and innate immunity, Biochem Biophys Res Commun, 388 (2009) 621-625.

[48] J.D. Schilling, S.M. Martin, C.S. Hung, R.G. Lorenz, S.J. Hultgren, Toll-like receptor 4 on stromal and hematopoietic cells mediates innate resistance to uropathogenic Escherichia coli, Proc Natl Acad Sci U S A, 100 (2003) 4203-4208.

[49] B. Ragnarsdottir, N. Lutay, J. Gronberg-Hernandez, B. Koves, C. Svanborg, Genetics of innate immunity and UTI susceptibility, Nat Rev Urol, 8 (2011) 449-468.

[50] A.J. Carey, M.J. Sullivan, B.L. Duell, D.K. Crossman, D. Chattopadhyay, A.J. Brooks, C.K. Tan, M. Crowley, M.J. Sweet, M.A. Schembri, G.C. Ulett, Uropathogenic Escherichia coli Engages CD14Dependent Signaling to Enable Bladder-Macrophage-Dependent Control of Acute Urinary Tract Infection, J Infect Dis, 213 (2016) 659-668.

[51] E. Andersen-Nissen, T.R. Hawn, K.D. Smith, A. Nachman, A.E. Lampano, S. Uematsu, S. Akira, A. Aderem, Cutting edge: Tlr5-/- mice are more susceptible to Escherichia coli urinary tract infection, J Immunol, 178 (2007) 4717-4720.

[52] M. Schiwon, C. Weisheit, L. Franken, S. Gutweiler, A. Dixit, C. Meyer-Schwesinger, J.M. Pohl, N.J. Maurice, S. Thiebes, K. Lorenz, T. Quast, M. Fuhrmann, G. Baumgarten, M.J. Lohse, G. Opdenakker, J. Bernhagen, R. Bucala, U. Panzer, W. Kolanus, H.J. Grone, N. Garbi, W. Kastenmuller, P.A. Knolle, C. Kurts, D.R. Engel, Crosstalk between sentinel and helper macrophages permits neutrophil migration into infected uroepithelium, Cell, 156 (2014) 456-468.

[53] J. Song, S.N. Abraham, Innate and adaptive immune responses in the urinary tract, Eur J Clin Invest, 38 Suppl 2 (2008) 21-28.

[54] A. Waldhuber, M. Puthia, A. Wieser, C. Cirl, S. Durr, S. Neumann-Pfeifer, S. Albrecht, F. Rommler, T. Muller, Y. Zheng, S. Schubert, O. Gross, C. Svanborg, T. Miethke, Uropathogenic Escherichia coli strain CFT073 disrupts NLRP3 inflammasome activation, J Clin Invest, 126 (2016) 2425-2436.

[55] J.W. Symington, C. Wang, J. Twentyman, N. Owusu-Boaitey, R. Schwendener, G. Nunez, J.D. Schilling, I.U. Mysorekar, ATG16L1 deficiency in macrophages drives clearance of uropathogenic E. coli in an IL-1beta-dependent manner, Mucosal Immunol, 8 (2015) 1388-1399.

[56] K. Schaale, K.M. Peters, A.M. Murthy, A.K. Fritzsche, M.D. Phan, M. Totsika, A.A. Robertson, K.B. Nichols, M.A. Cooper, K.J. Stacey, G.C. Ulett, K. Schroder, M.A. Schembri, M.J. Sweet, Strainand host species-specific inflammasome activation, IL-1beta release, and cell death in macrophages infected with uropathogenic Escherichia coli, Mucosal Immunol, 9 (2016) 124-136.

[57] N.J. Bokil, M. Totsika, A.J. Carey, K.J. Stacey, V. Hancock, B.M. Saunders, T. Ravasi, G.C. Ulett, M.A. Schembri, M.J. Sweet, Intramacrophage survival of uropathogenic Escherichia coli: differences between diverse clinical isolates and between mouse and human macrophages, Immunobiology, 216 (2011) 1164-1171.

[58] N. Owusu-Boaitey, K.A. Bauckman, T. Zhang, I.U. Mysorekar, Macrophagic control of the response to uropathogenic E. coli infection by regulation of iron retention in an IL-6-dependent manner, Immun Inflamm Dis, 4 (2016) 413-426.

[59] S. Yona, K.W. Kim, Y. Wolf, A. Mildner, D. Varol, M. Breker, D. Strauss-Ayali, S. Viukov, M. Guilliams, A. Misharin, D.A. Hume, H. Perlman, B. Malissen, E. Zelzer, S. Jung, Fate mapping reveals origins and dynamics of monocytes and tissue macrophages under homeostasis, Immunity, 38 (2013) 79-91. 
[60] Y. Lavin, D. Winter, R. Blecher-Gonen, E. David, H. Keren-Shaul, M. Merad, S. Jung, I. Amit, Tissue-resident macrophage enhancer landscapes are shaped by the local microenvironment, Cell, 159 (2014) 1312-1326.

[61] J. Helft, J. Bottcher, P. Chakravarty, S. Zelenay, J. Huotari, B.U. Schraml, D. Goubau, C. Reis e Sousa, GM-CSF Mouse Bone Marrow Cultures Comprise a Heterogeneous Population of CD11c(+)MHCII(+) Macrophages and Dendritic Cells, Immunity, 42 (2015) 1197-1211.

[62] M. Guilliams, B. Malissen, A Matter of Perspective: Moving from a Pre-omic to a SystemsBiology Vantage of Monocyte-Derived Cell Function and Nomenclature, Immunity, 44 (2016) 5-6. [63] D. Engel, U. Dobrindt, A. Tittel, P. Peters, J. Maurer, I. Gutgemann, B. Kaissling, W. Kuziel, S. Jung, C. Kurts, Tumor necrosis factor alpha- and inducible nitric oxide synthase-producing dendritic cells are rapidly recruited to the bladder in urinary tract infection but are dispensable for bacterial clearance, Infect Immun, 74 (2006) 6100-6107.

[64] E. Kolaczkowska, P. Kubes, Neutrophil recruitment and function in health and inflammation, Nat Rev Immunol, 13 (2013) 159-175.

[65] M. Haraoka, L. Hang, B. Frendeus, G. Godaly, M. Burdick, R. Strieter, C. Svanborg, Neutrophil recruitment and resistance to urinary tract infection, J Infect Dis, 180 (1999) 1220-1229.

[66] M.A. Ingersoll, K.A. Kline, H.V. Nielsen, S.J. Hultgren, G-CSF induction early in uropathogenic Escherichia coli infection of the urinary tract modulates host immunity, Cell Microbiol, 10 (2008) 2568-2578.

[67] J.M. Daley, A.A. Thomay, M.D. Connolly, J.S. Reichner, J.E. Albina, Use of Ly6G-specific monoclonal antibody to deplete neutrophils in mice, J Leukoc Biol, 83 (2008) 64-70.

[68] G. Godaly, A.E. Proudfoot, R.E. Offord, C. Svanborg, W.W. Agace, Role of epithelial interleukin-8 (IL-8) and neutrophil IL-8 receptor A in Escherichia coli-induced transuroepithelial neutrophil migration, Infect Immun, 65 (1997) 3451-3456.

[69] G. Godaly, L. Hang, B. Frendeus, C. Svanborg, Transepithelial neutrophil migration is CXCR1 dependent in vitro and is defective in IL-8 receptor knockout mice, J Immunol, 165 (2000) 5287-5294. [70] D. Hashimoto, A. Chow, C. Noizat, P. Teo, M.B. Beasley, M. Leboeuf, C.D. Becker, P. See, J. Price, D. Lucas, M. Greter, A. Mortha, S.W. Boyer, E.C. Forsberg, M. Tanaka, N. van Rooijen, A. Garcia-Sastre, E.R. Stanley, F. Ginhoux, P.S. Frenette, M. Merad, Tissue-resident macrophages selfmaintain locally throughout adult life with minimal contribution from circulating monocytes, Immunity, 38 (2013) 792-804.

[71] M. Saluja, P. Gilling, Intravesical bacillus Calmette-Guerin instillation in non-muscle-invasive bladder cancer: A review, Int J Urol, (2017).

[72] M. Babjuk, W. Oosterlinck, R. Sylvester, E. Kaasinen, A. Bohle, J. Palou-Redorta, M. Roupret, U. European Association of, EAU guidelines on non-muscle-invasive urothelial carcinoma of the bladder, the 2011 update, Eur Urol, 59 (2011) 997-1008.

[73] A.M. Jackson, A.B. Alexandroff, R.W. Kelly, A. Skibinska, K. Esuvaranathan, S. Prescott, G.D. Chisholm, K. James, Changes in urinary cytokines and soluble intercellular adhesion molecule-1 (ICAM-1) in bladder cancer patients after bacillus Calmette-Guerin (BCG) immunotherapy, Clin Exp Immunol, 99 (1995) 369-375.

[74] A. Bisiaux, N. Thiounn, M.O. Timsit, A. Eladaoui, H.H. Chang, J. Mapes, A. Mogenet, J.L. Bresson, D. Prie, S. Bechet, C. Baron, C. Sadorge, S. Thomas, E.B. Albert, P.S. Albert, M.L. Albert, Molecular analyte profiling of the early events and tissue conditioning following intravesical bacillus calmette-guerin therapy in patients with superficial bladder cancer, J Urol, 181 (2009) 1571-1580. [75] M.R. Saban, C. Simpson, C. Davis, G. Wallis, N. Knowlton, M.B. Frank, M. Centola, R.M. Gallucci, R. Saban, Discriminators of mouse bladder response to intravesical Bacillus CalmetteGuerin (BCG), BMC Immunol, 8 (2007) 6.

[76] G. Redelman-Sidi, M.S. Glickman, B.H. Bochner, The mechanism of action of BCG therapy for bladder cancer--a current perspective, Nat Rev Urol, 11 (2014) 153-162.

[77] H. Suttmann, J. Riemensberger, G. Bentien, D. Schmaltz, M. Stockle, D. Jocham, A. Bohle, S. Brandau, Neutrophil granulocytes are required for effective Bacillus Calmette-Guerin immunotherapy of bladder cancer and orchestrate local immune responses, Cancer Res, 66 (2006) 8250-8257.

[78] T.L. Ratliff, J.K. Ritchey, J.J. Yuan, G.L. Andriole, W.J. Catalona, T-cell subsets required for intravesical BCG immunotherapy for bladder cancer, J Urol, 150 (1993) 1018-1023. 
[79] C. Biot, C.A. Rentsch, J.R. Gsponer, F.D. Birkhauser, H. Jusforgues-Saklani, F. Lemaitre, C. Auriau, A. Bachmann, P. Bousso, C. Demangel, L. Peduto, G.N. Thalmann, M.L. Albert, Preexisting BCG-specific T cells improve intravesical immunotherapy for bladder cancer, Sci Transl Med, 4 (2012) 137ra172.

[80] H. Yamada, S. Matsumoto, T. Matsumoto, T. Yamada, U. Yamashita, Enhancing effect of an inhibitor of nitric oxide synthesis on bacillus Calmette-Guerin-induced macrophage cytotoxicity against murine bladder cancer cell line MBT-2 in vitro, Jpn J Cancer Res, 91 (2000) 534-542.

[81] Y. Luo, H. Yamada, D.P. Evanoff, X. Chen, Role of Th1-stimulating cytokines in bacillus Calmette-Guerin (BCG)-induced macrophage cytotoxicity against mouse bladder cancer MBT-2 cells, Clin Exp Immunol, 146 (2006) 181-188.

[82] Y. Luo, R. Han, D.P. Evanoff, X. Chen, Interleukin-10 inhibits Mycobacterium bovis bacillus Calmette-Guerin (BCG)-induced macrophage cytotoxicity against bladder cancer cells, Clin Exp Immunol, 160 (2010) 359-368.

[83] T. Hanada, M. Nakagawa, A. Emoto, T. Nomura, N. Nasu, Y. Nomura, Prognostic value of tumor-associated macrophage count in human bladder cancer, Int J Urol, 7 (2000) 263-269.

[84] H. Takayama, K. Nishimura, A. Tsujimura, Y. Nakai, M. Nakayama, K. Aozasa, A. Okuyama, N. Nonomura, Increased infiltration of tumor associated macrophages is associated with poor prognosis of bladder carcinoma in situ after intravesical bacillus Calmette-Guerin instillation, J Urol, 181 (2009) 1894-1900.

[85] C. Ayari, H. LaRue, H. Hovington, M. Decobert, F. Harel, A. Bergeron, B. Tetu, L. Lacombe, Y. Fradet, Bladder tumor infiltrating mature dendritic cells and macrophages as predictors of response to bacillus Calmette-Guerin immunotherapy, Eur Urol, 55 (2009) 1386-1395.

[86] L. Lima, D. Oliveira, A. Tavares, T. Amaro, R. Cruz, M.J. Oliveira, J.A. Ferreira, L. Santos, The predominance of M2-polarized macrophages in the stroma of low-hypoxic bladder tumors is associated with BCG immunotherapy failure, Urol Oncol, 32 (2014) 449-457.

[87] E.J. Askeland, M.R. Newton, M.A. O'Donnell, Y. Luo, Bladder Cancer Immunotherapy: BCG and Beyond, Adv Urol, 2012 (2012) 181987.

[88] C.L. Fu, J.I. Odegaard, D.R. Herbert, M.H. Hsieh, A novel mouse model of Schistosoma haematobium egg-induced immunopathology, PLoS Pathog, 8 (2012) e1002605.

[89] C.L. Fu, J.I. Odegaard, M.H. Hsieh, Macrophages are required for host survival in experimental urogenital schistosomiasis, FASEB J, 29 (2015) 193-207.

[90] T.A. Wynn, A. Chawla, J.W. Pollard, Macrophage biology in development, homeostasis and disease, Nature, 496 (2013) 445-455.

[91] J.S. Duffield, S.J. Forbes, C.M. Constandinou, S. Clay, M. Partolina, S. Vuthoori, S. Wu, R. Lang, J.P. Iredale, Selective depletion of macrophages reveals distinct, opposing roles during liver injury and repair, J Clin Invest, 115 (2005) 56-65.

[92] I. Goren, N. Allmann, N. Yogev, C. Schurmann, A. Linke, M. Holdener, A. Waisman, J. Pfeilschifter, S. Frank, A transgenic mouse model of inducible macrophage depletion: effects of diphtheria toxin-driven lysozyme M-specific cell lineage ablation on wound inflammatory, angiogenic, and contractive processes, Am J Pathol, 175 (2009) 132-147.

[93] M. Summan, G.L. Warren, R.R. Mercer, R. Chapman, T. Hulderman, N. Van Rooijen, P.P. Simeonova, Macrophages and skeletal muscle regeneration: a clodronate-containing liposome depletion study, Am J Physiol Regul Integr Comp Physiol, 290 (2006) R1488-1495.

[94] M.J. van Amerongen, M.C. Harmsen, N. van Rooijen, A.H. Petersen, M.J. van Luyn, Macrophage depletion impairs wound healing and increases left ventricular remodeling after myocardial injury in mice, Am J Pathol, 170 (2007) 818-829.

[95] S.T. Truschel, E. Wang, W.G. Ruiz, S.M. Leung, R. Rojas, J. Lavelle, M. Zeidel, D. Stoffer, G. Apodaca, Stretch-regulated exocytosis/endocytosis in bladder umbrella cells, Mol Biol Cell, 13 (2002) 830-846. 